$\begin{gathered}\text { Revista do Departamento de Geografia } \\ \text { Universidade de São Paulo } \\ \text { www.revistas.usp.br/rdg }\end{gathered}$
Volume Especial - XVII SBGFA / I CNGF (2017)

\title{
Solos e Evolução da Paisagem em Ambiente Periglacial na Península Barton, Antártica Marítima
}

\author{
Soils and Landscape Evolution in Periglacial Environment on the Barton \\ Peninsula, Maritime Antarctica
}

\author{
Davi do Vale Lopes \\ Universidade Federal de Minas Gerais \\ davivlopes@hotmail.com \\ José João Lelis Leal de Souza \\ Universidade Federal do Rio Grande do Norte \\ jjlelis@gmail.com
}

Fábio Soares de Oliveira

Universidade Federal de Minas Gerais fabiosolos@gmail.com

Carlos Ernesto G. R. Schaefer Universidade Federal de Viçosa

\begin{abstract}
Resumo: As baixas disponibilidade de água líquida e temperatura na Antártica condicionam a formação de solos incipientes. Condições climáticas menos severas na Antártica Marítima permitem o maior desenvolvimento de solos, com maior cobertura vegetal e intemperismo químico mais significativo. A Península Barton, localizada na Ilha Rei George (Antártica Marítima), possui solos derivados de litologias diversas, com destaque para o basalto/andesito piritizado, representando a área de maior extensão de materias sulfídicos na região. O objetivo deste trabalho foi analisar o papel do relevo na gênese de solos sulfatados e sua relação com a evolução da paisagem. Os solos foram classificados de acordo com a Soil Taxonomy e WRB/FAO. Foram descritos três perfis em toposequência e analisados seus atributos morfológicos, físicos, químicos e mineralógicos. A crioclastia está presente como um processo pedogenético, mas o que se destaca é o grau evolutivo dos solos associado à acidificação pela oxidação dos sulfetos. Esse processo promove a liberação de elementos como o $\mathrm{Fe}$, que são mobilizados por influência do relevo. Consequentemente, formam-se níveis encouraçados, isto é, horizontes petroplínticos, caracterizando uma transformação da cobertura pedológica. A gênese destes solos demonstra que a paisagem periglacial é dinâmica e de profundo interesse das investigações pedogeomorfológicas.
\end{abstract}

Palavras-chaves: Oxidação; Sulfetos; Pedogênese; Concreções; Pedogeomorfologia.
Abstract: Antarctic soils are characterized by low development degree due to low liquid water content. Compared to mainland, Maritime Antactica has a mild climate. These milder conditions allow a much greater development of soils and more significant chemical weathering. The study area is Barton Peninsula, the second largest ice-free area of King George Island (Maritime Antarctica). The Barton Peninsula has soils derived from different lithologies, with basalt / andesite (with pyrite) standing out. This study aimed to evaluate the role of relief in the genesis of sulfated soils. Three pedons were selected, described, sampled, and classified according to Soil Taxonomy and WRB/FAO. Morphological, physical, mineralogical and chemical analysis were performed. The Barton soils present polygenic evolution. The rock shattering caused by freezing and thawing is present as a pedogenetic process. However, the evolution degree of the soils is substantially associated with the acidification by sulphide oxidation. This process promotes the release of elements, such as $\mathrm{Fe}$, which are mobilized by the relief. Consequently, duricrusts (i.e. petroplintic horizons) are formed, characterizing a transformation of the pedological cover. The genesis of these soils shows that the peri-glacial landscape is dynamic and of deep interest in soil-geomorphological investigations.

Keywords: Oxidation; Sulfides; Pedogenesis;Concretions; Soil-Geomorphology. 


\section{INTRODUÇÃO}

A Antártica pode ser compartimentada em porção continental e marítima (BEYER E BÓLTER, 2002) e é conhecida como o continente dos superlativos: mais frio, mais ventoso, mais seco, mais alto, entre outros, ainda que tais condições não sejam homogêneas. Entre os arquipélagos que compõem a Antártica Marítima, as Shetlands do Sul possuem clima oceânico frio, com chuvas de verão frequentes e moderada amplitude térmica anual, varindo entre $8^{\circ} \mathrm{C}$ e $10^{\circ} \mathrm{C}$. A temperatura média anual é de aproximadamente $-3^{\circ} \mathrm{C}$. As áreas livres de gelo estão localizadas onde as temperaturas médias anuais excedem $-2^{\circ} \mathrm{C}$ (SERRANO E LOPEZ-MARTINEZ, 2000).

Condições climáticas menos severas na Antártica Marítima permitem o desenvolvimento de solos mais profundos, maior cobertura vegetal e intemperismo químico mais significativo em comparação com a Antártica Continental (CAMPBELL E CLARIDGE, 1987). A maior diversidade de processos repercute numa variedade de tipos de solos, com destaque para aqueles associados à dinamica do permafrost, os solos ornitogênicos e os solos sulfatados (FRANCELINO et al., 2011; MICHEL et al., 2014).

Nas Shetlands do Sul encontra-se Rei George, a maior ilha do Arquipélago. A Península Barton (PB) (62 $2^{\circ} 14^{\prime}$ S 58 $\left.46^{\prime} \mathrm{W}\right)$ localiza-se a sudoeste da Ilha Rei George, na Baía de Maxwell, e possui área aproximada de $12 \mathrm{~km}^{2}$. Nesta península é comum a presença de rochas enriquecidas em sulfetos que, uma vez expostos aos agentes intempéricos, são afetados por oxidação, criando condições favoráveis ao intemperismo geoquímico e culminando na gênese dos solos sulfatados.

Souza et al., (2014) registraram que na Ilha Seymour os solos ácidos sulfatados são os mais desenvolvidos. Na Península Keller, também Ilha Rei George, Simas et al., (2006) relataram presença de solos ácidos, horizontes sulfúricos, minerais de argila que indicam intemperismo químico mais avançado e desestabilização de minerais primários em áreas de oxidação de sulfetos. Ainda assim, poucos estudos abordam os solos sulfatados da Antártica e mais escassos ainda são os estudos que buscaram compreender o papel do relevo na sua gênese.

Face ao exposto, o objetivo deste estudo foi analisar, a partir de atributos fisicos e químicos, o papel do relevo na gênese de solos sulfatados na Península Barton e, da mesma maneira, como tais solos interagem com a evolução da paisagem em dominio peri-glacial da Antártica Marítima.

\section{MATERIAL E MÉTODOS}

\section{Área de Estudo}

A Península Barton localiza-se no arco Mesozoico-Cenozoico da Ilha Rei George, Arquipélago das Shetlands do Sul e compreende uma sequência terciária formada por lavas e rochas vulcanoclásticas (basáltica e andesíta), com intrusões de granodiorito e diorito na parte oeste (ARMSTRONG, 1995; YEO et al., 2004). A Formação Sejong, vulcanoclástica, é a mais antiga unidade geológica, possuindo afloramentos ao longo da costa (Figura 1). Além dela, a faixa costeira apresenta depósitos quaternários descontínuos em razão da presença dos corpos intrusivos.

A geomorfologia da área apresenta importantes distinções (LOPES-MARTINEZ et al., 2012). Nas porções central e oeste da Península (próximo à estação de pesquisa sul coreana King Sejong), os vales são mais amplos e os canais de drenagem mais organizados. Ao norte, próxima à geleira, o relevo é mais escarpado. As plataformas médias (16 - 60 metros de amplitude altimétrica) ocorrem principalmente ao sul da Península, voltadas para a Baía de Maxwell e sob a Formação Sejong. As plataformas mais elevadas (61 105 metros de amplitude altimétrica) são mais descontínuas, com muitos corpos plutônicos intercalados, desenhando escarpas íngremes. Áreas de depósitos marinhos elevados (106 - 260 metros de amplitude altimétrica) coincidem com os dois principais campos de solos poligonais. Entre os picos mais elevados, destaca-se Noel Hill (Baekdubong). 


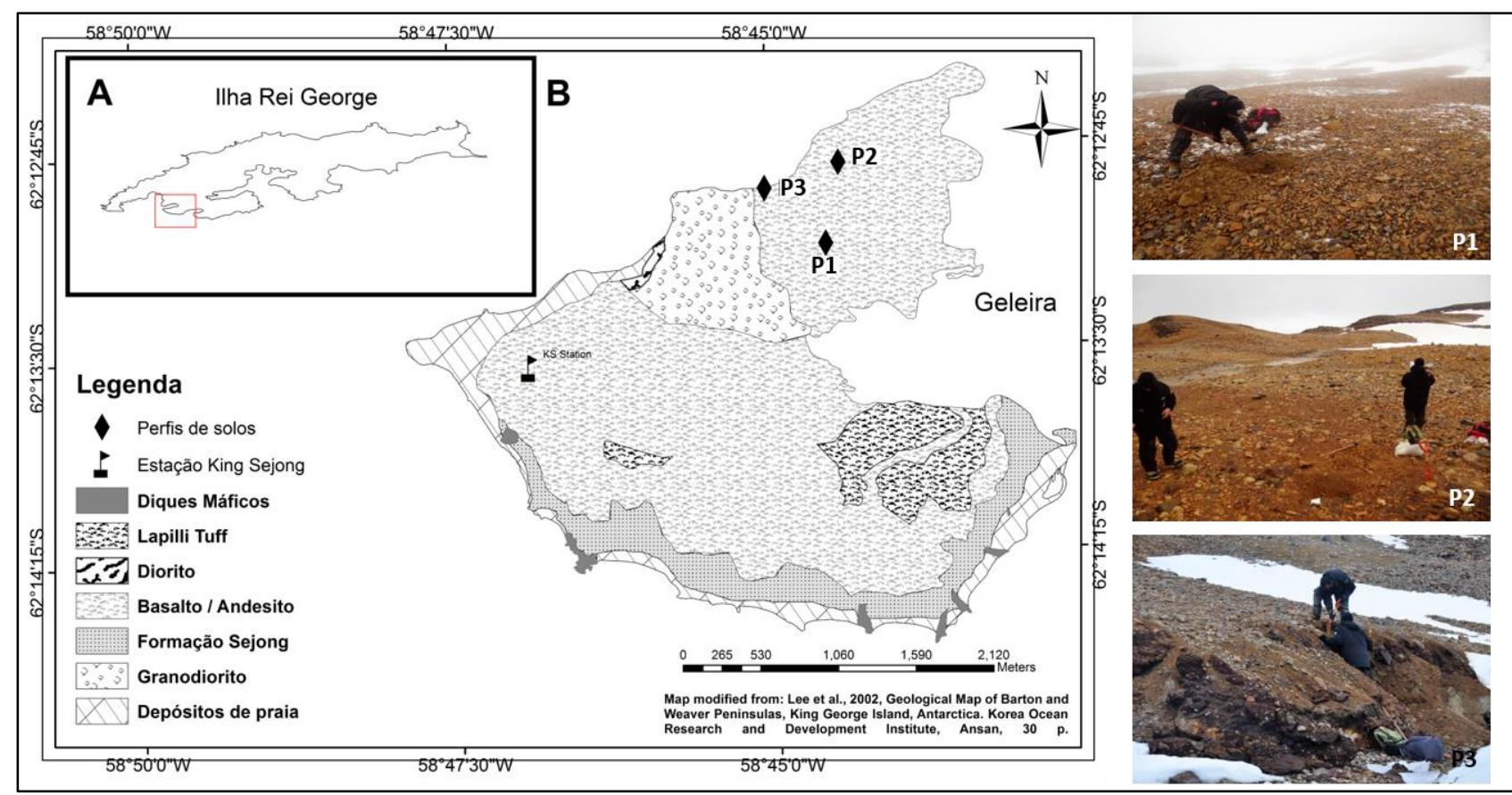

Figura 1: A - Mapa de localização da Península Barton na Ilha Rei George, Antártica Marítima. B - Mapa litológico da Península Barton com a indicação da localização dos perfis de solos amostrados e imagens ilustrativas dos respectivos ambientes.

\section{Trabalho De Campo, Coleta e Caracterização dos Solos}

O estudo envolveu a seleção de uma vertente com uniformidade litológica e onde a presença de solos sulfatados fosse verificada. Armstrong (1995) identificou que na porção norte ocorre abundante associação de sulfetos com andesitos e, por isso, essa área foi priorizada para investigação. Após reconhecimento em campo, foi selecionada uma topossequência, onde três trincheiras foram abertas, respectivamente na alta (P1), média (P2) e baixa encosta (P3) (Tabela 1).

Foram descritos os perfis das faces a montante e amostras deformadas foram coletadas. Após transporte, as amostras foram destorroadas, secas e peneiradas (malha de $2 \mathrm{~mm}$ ). Separou-se as frações granulométricas pelo método da centrífuga com uso de solução de $\mathrm{Na}_{2} \mathrm{CO}_{3} \mathrm{em} \mathrm{pH} \mathrm{9,5} \mathrm{como} \mathrm{dispersante.}$

$\mathrm{O} \mathrm{pH}$, nutrientes trocáveis e textura foram determinados em amostras de terra fina seca ao ar (TFSA) (EMBRAPA, 1997). Cátions trocáveis, $\mathrm{Ca}^{2+}, \mathrm{Mg}^{2+}$ e $\mathrm{Al}^{3+}$ foram extraídas com $1 \mathrm{M} \mathrm{KCl} \mathrm{e} \mathrm{P}, \mathrm{Na}^{+}$e $\mathrm{K}^{+}$com extrator Mehlich-1 (dupla diluição 0,05 mol/L de $\mathrm{HCl}$ em 0,0125 mol/L de $\mathrm{H}_{2} \mathrm{SO}_{4}$ ) (EMBRAPA, 1997). Os teores dos elementos $\left(\mathrm{Al}^{3+}, \mathrm{Ca}^{2+} \mathrm{e} \mathrm{Mg}^{2+}\right)$ nos extratos foram determinados por espectrometria de absorção atômica e emissão de chama $\left(\mathrm{Na}^{+}\right.$e $\mathrm{K}^{+}$) e fotocolorimetria $(\mathrm{P})$. $\mathrm{O}$ carbono orgânico total foi determinado via combustão úmida (YEOMANS E BREMNER, 1988). Os solos foram classificados de acordo com a Soil Taxonomy (SSS, 2014) e World Reference Base for Soil Resources - WRB (IUSS, 2014).

A mineralogia foi analisada no Laboratório Nacional de Luz Síncroton (LNLS), em Campinas-SP. A linha utilizada foi X-ray powder diffraction (XPD), dedicada a materiais policristalinos. Utilizou-se o equipamento de difração de raios-X HUBER, no modo de operação de alta resolução. Os resultados foram interpretados através de padrões de literatura (BRINDLEY E BROWN, 1980). 
Tabela 1: Aspectos gerais dos perfis que compõem a topossequência estudada na Península Barton.

\begin{tabular}{|c|c|c|c|c|c|c|}
\hline Perfil & $\begin{array}{c}\text { Altitude } \\
\text { (m) }\end{array}$ & $\begin{array}{l}\text { Coordenadas } \\
\text { Geográficas }\end{array}$ & Descrição Geral & $\begin{array}{c}\text { Soil } \\
\text { Taxonomy } \\
\end{array}$ & WRB/FAO & Imagem \\
\hline $\mathrm{P} 1$ & 160 & $\begin{array}{c}0409394 / \\
3100246\end{array}$ & $\begin{array}{l}\text { Relevo plano, porção } \\
\text { superior da } \\
\text { topossequência. Área } \\
\text { extensa e representativa } \\
\text { da parte mais elevada da } \\
\text { plataforma. Observou-se } \\
\text { rochas quebradas, porém } \\
\text { em profundidade } \\
\text { encontra-se raior } \\
\text { presença de partículas } \\
\text { finas. Os fragmentos são } \\
\text { muito amarelados. Não } \\
\text { se observou água } \\
\text { minando no perfil. }\end{array}$ & $\begin{array}{c}\text { Typic } \\
\text { dystrocryepts }\end{array}$ & $\begin{array}{l}\text { Haplic Leptic } \\
\text { Cambisol }\end{array}$ & \\
\hline P2 & 83 & $\begin{array}{c}0409476 / \\
3100799\end{array}$ & $\begin{array}{l}\text { Relevo suave ondulado, } \\
\text { porção média da } \\
\text { topossequência. } \\
\text { Superfície com coloração } \\
\text { extremamente } \\
\text { amarelada. O perfil } \\
\text { apresentava mosqueados. } \\
\text { Ausência de cobertura } \\
\text { vegetal. Canais de } \\
\text { drenagem próximos } \\
\text { indicando drenagem } \\
\text { superficial } \\
\text { subsuperficial. }\end{array}$ & $\begin{array}{c}\text { Typic } \\
\text { sulfaquepts }\end{array}$ & $\begin{array}{c}\text { Hyperthionic } \\
\text { Leptic } \\
\text { Cambisol }\end{array}$ & \\
\hline $\mathrm{P} 3$ & 10 & $\begin{array}{c}0408974 / \\
3100616\end{array}$ & $\begin{array}{l}\text { Relevo declivoso, porção } \\
\text { inferior ou baixa da } \\
\text { topossequência. O perfil } \\
\text { está no contato com o } \\
\text { domínio litorâneo, } \\
\text { próximo as águas da } \\
\text { Marian Cove. Presença } \\
\text { de horizonte } \\
\text { petroplíntico. Ausência } \\
\text { de cobertura vegetal. }\end{array}$ & $\begin{array}{l}\text { "Sulfuric" } \\
\text { petraquepts }\end{array}$ & $\begin{array}{c}\text { Orthothionic } \\
\text { Petroplinthic } \\
\text { Cambisol }\end{array}$ & \\
\hline
\end{tabular}

\section{RESULTADOS}

Os resultados das análises fisicas e químicas são apresentados, respectivamente, nas Tabelas 2 e 3. Os solos das porções alta e média da topossequência são mais homogêneos em termos de cor e apresentaram predomínio de matiz 10YR na maior parte dos horizontes, bem como altos valores de croma (Tabela 2). Alguns horizontes na base de P3, na porção baixa, apesentaram matiz mais avermelhada (2,5YR) intercalada com zonas acinzentadas e amareladas, revelando um solo de coloração variegada, sobreposto por um nivel concrecionado (Csm). O predominio de cores amareladas é semelhante ao que foi observado em outros solos sulfatados (FRANCELINO et al., 2011; SIMAS et al., 2006; SOUZA et al., 2014) e, tal como nestes estudos, solos com essa coloração são mais argilosos que outros solos da Antártica . Tais características corroboram com a indicação de Campbell \& Claridge (1987) da importância de considerar a cor como um atributo fundamental no estudo de solos em dominios polares, inclusive do seu grau evolutivo.

De maneira geral, os solos situados na porção alta da topossequência são mais argilosos nos horizontes superiores. Nas porções média e baixa essa relação se inverte, com maior conteúdo de argila em profundidade (Tabela 2). A presença do nível concrecionario parece exercer uma influência na textura destes solos. A classe textural variou de franco-argilosa, franco-argilo-siltosa a franco-arenosa nas porções alta e média e franco-arenosa a areia na porção baixa. 
Tabela 2: Atributos morfológicos e fisicos dos solos da topossequência

\begin{tabular}{|c|c|c|c|c|c|c|c|c|c|c|c|}
\hline Hor. & Prof. & AG & $\mathbf{A F}$ & Silte & Argila & $\mathbf{S}+\mathbf{A}$ & Classe Textural & Cor $(\operatorname{seco})$ & Nome (seco) & Cor (úmida) & Nome (úmida) \\
\hline \multicolumn{12}{|c|}{ P1 - Typic Dystrocryepts (Haplic Leptic Cambisol) } \\
\hline A & $0-5 / 7$ & 33 & 19 & 26 & 22 & 48 & Franco-Argilo-Arenosa & 10YR 8/4 & very pale brown & 10YR 5/6 & yellowish brown \\
\hline B1 & $5 / 7-22 / 52+$ & 45 & 11 & 22 & 22 & 44 & Franco-Argilo-Arenosa & 10YR 8/6 & yellow & 10YR 5/8 & yellowish brown \\
\hline $\mathrm{B} 2$ & $21-33$ & 51 & 17 & 17 & 15 & 32 & Franco-Arenosa & 10YR 8/4 & very pale brown & 10YR 3/4 & dark yellowish brown \\
\hline B3 & $33-51+$ & 64 & 8 & 10 & 18 & 28 & Franco-Arenosa & 10YR 7/6 & yellow & 10YR 5/8 & yellowish brown \\
\hline \multicolumn{12}{|c|}{ P2 - Typic Sulfaquepts (Hyperthionic Leptic Cambisol) } \\
\hline $\mathrm{Aj}$ & $0-10$ & 46 & 17 & 18 & 19 & 37 & Franco-Arenosa & 10YR 6/8 & yellow & 10YR 5/6 & yellowish brown \\
\hline Bjv & $10-25+$ & 31 & 10 & 29 & 30 & 59 & Franco-Argilosa & $\begin{array}{l}\text { 10YR 7/8 } \\
\text { 10YR 8/6 }\end{array}$ & $\begin{array}{l}\text { yellow } \\
\text { Yellow }\end{array}$ & $\begin{array}{l}10 Y R 5 / 8 \\
10 Y R 7 / 6\end{array}$ & yellowish brown yellow \\
\hline \multicolumn{12}{|c|}{ P3 - "Sulfuric" Petraquepts (Orthothionic Petroplinthic Cambisol) } \\
\hline A & $0-20 / 29$ & 60 & 14 & 13 & 13 & 26 & Franco-Arenosa & 10YR 7/4 & very pale brown & 10YR 4/6 & dark yellowish brown \\
\hline $\mathrm{Bj}$ & 20/29-36/40 & 89 & 4 & 3 & 4 & 7 & Areia & $5 \mathrm{YR} 5 / 6$ & yellowish red & $2.5 \mathrm{YR} 2,5 / 3$ & dark reddish brown \\
\hline Csm & 36/40-90/94 & - & - & - & - & - & - & - & - & - & - \\
\hline $2 \mathrm{Bj} 1$ & 90/94-130 & 69 & 6 & 13 & 12 & 25 & Franco-Arenosa & 10YR 8/3 & yellow pale brown & 10YR 4/6 & dark yellowish brown \\
\hline $2 \mathrm{Bj} 2$ & $130-140 / 165$ & 13 & 41 & 27 & 19 & 46 & Franco-Arenosa & Gley $18 / 10 \mathrm{Y}$ & light greenish gray & Gley $17 / 10 Y$ & light greenish gray \\
\hline 2Bjv3 & $140 / 165-220+$ & 40 & 29 & 14 & 17 & 31 & Franco-Arenosa & $\begin{array}{c}2,5 \text { YR } 8 / 4 \\
10 Y R ~ 8 / 4 \\
\end{array}$ & $\begin{array}{c}\text { pale yellow } \\
\text { very pale brown }\end{array}$ & $\begin{array}{c}5 \mathrm{Y} 7 / 3 \\
10 \mathrm{YR} 4 / 6 \\
\end{array}$ & $\begin{array}{l}\text { pale yellow dark } \\
\text { yellowish brown }\end{array}$ \\
\hline
\end{tabular}

Tabela 3: Atributos químicos dos solos da topossequência

\begin{tabular}{|c|c|c|c|c|c|c|c|c|c|c|c|c|c|c|c|c|c|c|c|c|c|}
\hline \multirow{2}{*}{ Hor. } & pH & $\mathbf{P}$ & $\mathbf{K}$ & $\mathbf{N a}$ & $\mathrm{Ca}^{2+}$ & $\mathrm{Mg}^{2+}$ & $\mathbf{A l}^{3+}$ & $\mathbf{H}+\mathbf{A l}$ & SB & $\mathbf{t}$ & $\mathbf{T}$ & $\mathbf{V}$ & $\mathbf{m}$ & CO & P-Rem & $\mathbf{C u}$ & Mn & $\mathbf{F e}$ & $\mathbf{Z n}$ & $\mathbf{N i}$ & $\mathbf{P b}$ \\
\hline & $\mathrm{H}_{2} \mathrm{O}$ & \multicolumn{3}{|c|}{ mg.dm ${ }^{-3}$} & \multicolumn{7}{|c|}{ cmolc. $\mathrm{dm}^{-3}$} & \multicolumn{2}{|c|}{$\%$} & dag. $\mathrm{Kg}^{-1}$ & mg.L. $\mathrm{L}^{-1}$ & \multicolumn{6}{|c|}{$\mathrm{mg} \mathrm{dm}^{3}$} \\
\hline \multicolumn{22}{|c|}{ P1 - Typic Dystrocryepts (Haplic Leptic Cambisol) } \\
\hline A & 5.44 & 6.6 & 35 & 78.8 & 3.1 & 0.81 & 1.7 & 4 & 4.34 & 6.04 & 8.34 & 52.0 & 28.1 & 0.13 & 27.4 & 2.19 & 14.6 & 482.3 & 1.79 & 0.68 & 1.3 \\
\hline B1 & 4.64 & 7.3 & 13 & 26.5 & 2.77 & 0.39 & 2.6 & 6.3 & 3.31 & 5.91 & 9.61 & 34.4 & 44 & 0.26 & 14.6 & 0.7 & 1.5 & 195.7 & 0.69 & 0.36 & 2.01 \\
\hline B2 & 5.49 & 9.8 & 27 & 99.0 & 3.96 & 0.87 & 0.4 & 3.5 & 5.33 & 5.73 & 8.83 & 60.4 & 7 & 0.26 & 22.5 & 3.35 & 14.9 & 630.2 & 3.55 & 0.6 & 0.36 \\
\hline B3 & 4.68 & 7.1 & 13 & 36.5 & 0.48 & 0.55 & 1.5 & 4.8 & 1.22 & 2.72 & 6.02 & 20.3 & 55.1 & 0.13 & 23.8 & 1.57 & 4.9 & 305.7 & 2.13 & 0.55 & 2.52 \\
\hline \multicolumn{22}{|c|}{ P2 - Typic Sulfaquepts (Hyperthionic Leptic Cambisol) } \\
\hline $\mathrm{Aj}$ & 3.21 & 5.1 & 1 & 32.5 & 3.26 & 0.38 & 6.9 & 15.5 & 3.78 & 10.68 & 19.28 & 19.6 & 64.6 & 0.26 & 10.8 & 1.83 & 2.8 & 815 & 0.44 & 0.83 & 0 \\
\hline Bjv & 3.1 & 11 & 0 & 34.5 & 3.4 & 0.32 & 6.6 & 12.4 & 3.87 & 10.47 & 16.27 & 23.8 & 63 & 0.9 & 7.4 & 2.21 & 2.7 & 899.5 & 0.49 & 0.76 & 0 \\
\hline \multicolumn{22}{|c|}{ P3 - “Sulfuric” Petraquepts (Orthothionic Petroplinthic Cambisol) } \\
\hline A & 4.25 & 10.7 & 13 & 70.8 & 2.62 & 0.14 & 5.1 & 9.2 & 3.1 & 8.2 & 12.3 & 25.2 & 62.2 & 0.13 & 11.6 & 4.59 & 2.3 & 628.4 & 0.49 & 0.51 & 0 \\
\hline $\mathrm{Bj}$ & 3.99 & 2.9 & 3 & 26.5 & 2.79 & 0.12 & 2.7 & 7.8 & 3.03 & 5.73 & 10.83 & 28.0 & 47.1 & 0.13 & 20.7 & 1.66 & 1.1 & 678 & 0.26 & 0.58 & 0 \\
\hline Csm & - & - & - & - & - & - & - & - & - & - & - & - & - & - & - & - & - & - & - & - & - \\
\hline $2 \mathrm{Bj} 1$ & 3.9 & 18.8 & 13 & 54.7 & 2.99 & 0.13 & 4.8 & 9.7 & 3.39 & 8.19 & 13.09 & 25.9 & 58.6 & 0.26 & 16.6 & 3.84 & 2.9 & 262.1 & 0.58 & 0.77 & 0 \\
\hline $2 \mathrm{Bj} 2$ & 3.55 & 80.3 & 7 & 14.4 & 2.58 & 0.14 & 5.1 & 9.2 & 2.8 & 7.9 & 12 & 23.3 & 64.6 & 0.13 & 17 & 0.7 & 1.2 & 342.3 & 0.21 & 0.59 & 0 \\
\hline 2Bjv3 & 3.3 & 82.8 & 0 & 14.4 & 2.67 & 0.16 & 3.5 & 10.6 & 2.89 & 6.39 & 13.49 & 21.4 & 54.8 & 0.13 & 8 & 0.57 & 0.9 & 1208.1 & 0.26 & 0.93 & 0 \\
\hline
\end{tabular}


Quimicamente, embora os solos possam ser majoritariamente descritos em toda a topossequência como pobres em carbono orgânico, distróficos, com alta saturação por alumínio e ácidos (Tabela 3), algumas diferenças entre as distintas posições devem ser destacadas. Os solos das porções média e baixa apresentam menores valores de $\mathrm{pH}$, quase sempre inferior a 4 , bem como maiores valores de saturação por alumínio. Na saturação por bases, apenas em dois horizontes de P1 os valores foram superiores a 50\%, condizentes com os maiores valores de $\mathrm{pH}$ encontrados. Sobre os micronutrientes, os solos apresentam teores elevados de $\mathrm{Fe}$, principalmente na porção baixa. $\mathrm{O}$ mesmo acontece com o $\mathrm{Cu}\left(4,59 \mathrm{mg} \cdot \mathrm{dm}^{3}\right)$ e $\mathrm{Ni}$ $\left(0,93 \mathrm{mg} \cdot \mathrm{dm}^{3}\right)$. Na porção alta foram verificados os maiores valores de $\mathrm{Mn}\left(14,6 \mathrm{mg} \cdot \mathrm{dm}^{3}\right)$ e $\mathrm{Pb}(2,52$ mg.dm³ (Tabela 3).

Mineralogicamente, os três solos da topossoquência são semelhantes. O difratogramas dos horizontes $\mathrm{Aj}$ e Bjv de P2 são apresentados como representativos (Figura 2). Foram identificados picos de minerais secundários como goethita $(2,21 ; 2,42 ; 2,61 \AA$ A) e natrojarosita $(2,76 ; 3,06 ; 3,12 ; 5,98 \AA$ ). Os picos 1,$46 ; 1,62$; 2,$46 ; 2,58 \AA$ sugerem a presença de maghemita. Em relação às fases minerais primárias, foram identificados picos da clorita $(1,56 ; 2,82 ; 7,98 \AA)$ ), feldspato $(3,72 ; 3,95 ; 3,96 ; 6,23 ; 6,60 \AA)$, magnetita $(1,44 ; 1,60 ; 2,90$; $2,97 \AA$ ) e pirita $(1,64 ; 1,89 ; 2,25 \AA)$. Essa paragênese condiz com a gênese dos solos associada aos andesitos ricos em sulfetos.

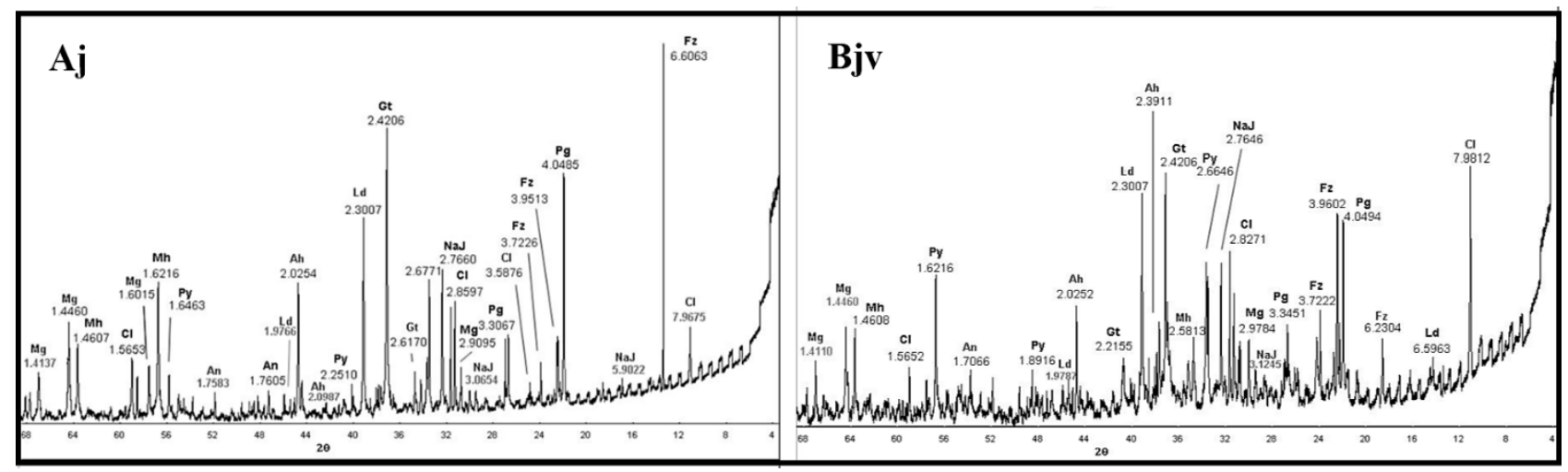

Figura 2: DRX da fração argila dos horizontes Aj e Bjv de P2, representativo da mineralogia dos solos da topossequência. An=Alunita, $\mathrm{Ah}=$ Anidrita, $\mathrm{Cl}=\mathrm{Clorita}$. $\mathrm{Fz}=$ Feldspato, $\mathrm{Gt}=$ Goethita, Ld=Lepidocrocita, $\mathrm{Mg}=$ Magnetita, $\mathrm{Mh}=$ Maghemita, NaJ=Natrojarosita, $\mathrm{Pg}=$ Plagioclásio, $\mathrm{Py}=$ Pirita.

P1 foi classificado como Typic dystrocryepts (SSS, 2014) ou Haplic Leptic Cambisol (IUSS, 2014). Embora a superfície seja pedregosa, no interior do perfil, como visto, o conteúdo de partículas finas (silte + argila) chama atenção. P2 foi classificado como Typic sulfaquepts (SSS, 2014) ou Hyperthionic Leptic Cambisol (IUSS, 2014). Apresenta horizonte sulfuric ( $\mathrm{pH}$ entre 3,5 e 4,0) dentro de $50 \mathrm{~cm}$, estando sua condição ácida relacionada à alta saturação por alumínio observada. P3, por fim, foi classificado como "Sulfuric" petraquepts (SSS, 2014) ou Orthothionic Petroplinthic Cambisol (IUSS, 2014). O pH inferior a 4 sugere a indicação do horizonte sulfuric, o que favorece a maior mobilidade iônica. Possui contato lítico dentro de $50 \mathrm{~cm}$ e a camada de concreção entre $38-92 \mathrm{~cm}$ é continua, denotando a presença de horizonte petroplíntico.

\section{DISCUSSÃO}

Tanto do ponto de vista morfológico quanto analítico, os resultados mostram a inegável influência do material de origem na gênese dos solos estudados, considerando que a presença dos sulfetos e a maneira como os mesmos são alterados responde por grande parte dos atributos observados. Contudo, os resultados mostram também um importante papel do relevo, principalmente no que se refere à dinâmica e mobilidade dos constituintes, resultante na formação de distintas feições conforme a posição na paisagem. Os dois aspectos serão discutidos a seguir.

Considerando o $\mathrm{pH}$ dos solos analisados, toda a cobertura pedológica pode ser entendida como "solos ácidos sulfatados". Ocorrem valores de $\mathrm{pH}$ inferior a 4,0, acompanhados por elevados valores de $\mathrm{Al}$ trocável e coloração amarelada, indicadora da presença de minerais sulfatados e óxidos; confirmada na análise mineralógica. Comparado aos estudos desenvolvidos por Simas et al. (2008) e Souza et al. (2014), alguns horizontes identificados neste estudo estão entre os mais ácidos já registrados na Antártica (Tabela 
4). Essa acidez gerada por frentes de sulfurização criou as condições necessárias para o intemperismo geoquímico que, associado a crioclastia intensa, foram responsáveis pela gênese destes solos.

Tabela 4: Valores de $\mathrm{pH}$ em $\mathrm{H}_{2} \mathrm{O}$ de solos ácidos sulfatados da Antártica Marítima.

\begin{tabular}{|c|c|c|c|c|c|c|}
\hline Dados & Local & $\begin{array}{l}\text { Id do } \\
\text { perfil }\end{array}$ & Hor. & $\begin{array}{l}\text { Prof. } \\
(\mathrm{cm})\end{array}$ & $\mathbf{p H}$ & Soil Taxonomy / WRB-FAO \\
\hline $\begin{array}{l}\text { Simas et al., } \\
\quad 2008\end{array}$ & \multirow{2}{*}{$\begin{array}{l}\text { Península Keller, Ilha Rei } \\
\text { George }\end{array}$} & K24-SH & $\mathrm{AC}$ & $0-20$ & 5.0 & Sulphuric Haploturbel \\
\hline $\begin{array}{l}\text { Simas et al., } \\
\quad 2008\end{array}$ & & K24-SH & $\mathrm{C}$ & $20-60$ & 4.4 & Sulphuric Haploturbel \\
\hline \multirow{10}{*}{$\begin{array}{l}\text { Souza et al., } \\
\quad 2014\end{array}$} & \multirow{10}{*}{ Ilha Seymour } & $\mathrm{P} 4$ & $\mathrm{Aj}$ & $0-5$ & 5.0 & "Sulfuric" Anhyorthel/“"Thionic" Cryosol \\
\hline & & P4 & $\mathrm{Cj} 1$ & $35-40$ & 3.2 & "Sulfuric" Anhyorthel/"Thionic" Cryosol \\
\hline & & P4 & $\mathrm{Cj} 2$ & $40-55$ & 3.1 & "Sulfuric" Anhyorthel//Thionic" Cryosol \\
\hline & & $\mathrm{P} 4$ & $\mathrm{Cj} 3$ & $55-88$ & 3.5 & "Sulfuric" Anhyorthel/"Thionic" Cryosol \\
\hline & & P7 & $\mathrm{Cj}$ & $8-38$ & 3.6 & Sulfuric Aquiturbel/Oxyaquic-Gelic Cryosol \\
\hline & & $\mathrm{P} 18$ & Cfj2 & $55 / 60-100$ & 3.3 & Sulfuric Aquorthel/“"Thionic”-Haplic Cryosol \\
\hline & & P19 & $\mathrm{Cj} 1$ & $\begin{array}{c}30 / 35- \\
55 / 60\end{array}$ & 3.3 & "Sulfuric" Haploturbel/“Thionic"-Turbi Cryosol \\
\hline & & $\mathrm{P} 20$ & Cjf2 & $25 / 60-100$ & 3.6 & "Sulfuric" Haploturbel/“Thionic"-Turbi Cryosol \\
\hline & & $\mathrm{P} 21$ & Crj1 & $\begin{array}{l}50 / 55- \\
70 / 75\end{array}$ & 3.7 & "Sulfuric" Anhyorthel/“Thionic"-Salic Cryosol \\
\hline & & $\mathrm{P} 21$ & Crjf2 & $70 / 75-120$ & 3.0 & "Sulfuric" Anhyorthel/“Thionic"-Salic Cryosol \\
\hline \multirow{4}{*}{$\begin{array}{l}\text { Presente } \\
\text { estudo }\end{array}$} & \multirow{4}{*}{$\begin{array}{c}\text { Península Barton, Ilha Rei } \\
\text { George }\end{array}$} & $\mathrm{P} 2$ & $\mathrm{Aj}$ & $0-10$ & $\begin{array}{c}3.2 \\
1\end{array}$ & Typic sulfaquepts / Hyperthionic Leptic Cambisol \\
\hline & & $\mathrm{P} 2$ & Bjv & $10-25$ & 3.1 & Typic sulfaquepts / Hyperthionic Leptic Cambisol \\
\hline & & $\mathrm{P} 1$ & $2 \mathrm{~B} 2$ & $\begin{array}{c}130- \\
140 / 165\end{array}$ & $\begin{array}{c}3.5 \\
5\end{array}$ & $\begin{array}{c}\text { "Sulfuric" Placic petraquepts / Orthothionic } \\
\text { Petroplinthic Cambisol }\end{array}$ \\
\hline & & $\mathrm{P} 1$ & $2 \mathrm{~B} 3$ & $\begin{array}{l}140 / 165- \\
220+\end{array}$ & 3.3 & $\begin{array}{l}\text { "Sulfuric" Placic petraquepts / Orthothionic } \\
\text { Petroplinthic Cambisol }\end{array}$ \\
\hline
\end{tabular}

Num estudo na mesma peninsula, Lee et al. (2004) havia destacado o baixo grau de intemperismo dos solos, mesmo daqueles em áreas de rochas com sulfetos. Os resultados aqui obtidos sinalizam o contrário, pois é fundamental que os atributos dos solos sejam avaliados considerando as caracteristicas do ambiente antártico. Assim, embora em termos taxonômicos ocorra uma cobertura pedológica cambissólica em toda a topossequência, o que, para as condições tropicais denotaria sua jovialidade, para o contexto antártico a presença de horizonte $\mathrm{B}$, ainda que incipiente, sinaliza um avançado grau evolutivo. A profundidade dos solos, sua mineralogia, atributos químicos e físicos reiteram essa constatação, corroborando com os estudos que incluem os solos sulfatados como exemplos de uma das maiores expressões do intemperismo químico na região.

Sobre a influência do relevo na gênese destes solos, os atributos sugerem que a variação altimétrica não representa o componente controlador do grau evolutivo, de maneira que na posição mais alta da topossequência os solos sejam mais evoluidos que na posição mais baixa. Ao contrário, tendo o pH, $\mathrm{m} \mathrm{e} \mathrm{V}$ como indicadores, o que se observa é que os solos das porções mediana e baixa podem ser considerados como mais intemperizados, o que está relacionado à dinâmica de movimentação das soluções ácidas.

A acidificação gerada pela oxidação dos sulfetos tem um papel importante no intemperismo mineral e solubilização de consituintes químicos, como o Fe e outros íons (FRANCELINO et al., 2011). Contudo, o relevo exerce importante influência na dinâmica de transporte de soluções e mobilização desse Fe (no perfil e na vertente). As concreções localizadas na base da topossequência sugerem isso, indicando que sua formação está relacionada a uma dinâmica pedológica mais complexa que aquela até entao considerada para o ambiente antártico. Na base da vertente, por exemplo, é preciso dimensionar a influência do spay salino e da própria maré na criação de condições favoráveis à precipitação do $\mathrm{Fe}$, elevando o $\mathrm{pH}$ e contribuindo com a formação do pavimento concrecionário. De outra maneira, a oxidação de sulfetos por si só já oportuniza a neoformação de hidroxidos de ferro, tal como descrito na gênese de gossans (TAYLOR \& EGGLETON, 2001). Quaisquer que sejam os mecanismos que expliquem as ocorrências observadas em Barton, tema de pesquisa em execução, há que se considerar que as variações observadas na topossequência sinalizam para o importante papel do relevo na gênese e evolução dos solos de áreas peri-glaciais. 
A gênese de concreções ferruginosas constituindo horizontes encouraçados, ou petroplínticos, está geralmente associada a dois conjuntos de processos. O primeiro seria a acumulação relativa de ferro através do intemperismo in situ de rochas, onde essa concentração ocorreria a partir da perda geoquímica dos demais constituintes. A segunda, por sua vez, seria pelo enriquecimento absoluto de ferro em áreas de descarga do lençol freático (Goudie, 1973; McFarlane, 1976), no qual o ferro é transportado pelas águas subterrâneas em solução e/ou como quelatos e se acumula em ambientes hidromórficos, bordas de planaltos, sopés de vertentes, etc (Tardy, 1993). Em ambos, contudo, as condições climáticas tropicais são sempre destacadas, com climas úmidos a semi-úmidos apontados como fundamentais.

É fato que os processos de laterização demonstram sua maior expressão nas regiões tropicias e produziram e/ou produzem nelas seus materiais mais característicos. Entretanto, sob condições muito particulares, como as observadas neste estudo, materiais semelhantes podem ser produzidos em outros contextos ambientais, como a Antártica. Para isso uma combinação de fatores, dentro o quais se destaca o papel do relevo, foi fundamental. Esse cenário abre possibilidades para a interpretação dos processos pedológicos na Antártica como nunca antes, suscitando pesquisas sobre as transformações pedológicas numa concepção biogeodinâmica (BOCQUIER, 1973) e ampliando os conhecimentos e relação destes com a evolução da paisagem no domínio peri-glacial.

\section{CONCLUSÕES}

A evolução da paisagem na Península Barton está diretamente ligada à combinação de processos de intemperismo físico, como a crioclastia, já amplamente conhecido e discutido para os solos da Antártica, mas também ao intemperismo químico acentuado. Em áreas com rochas sulfetadas, a exposição dos sulfetos gera oxidação e promove a acidificação dos solos, mobilizando constituintes que, associados ao papel do relevo, criam feições e produtos sui generis, transformando a cobertura pedológica.

As principais propriedades nos solos influenciadas pela oxidação de sulfetos foram cor, textura e $\mathrm{pH}$. Estas propriedades indicam presença generalizada de sulfatos e óxidos, confirmados pelas análises mineralógicas e texturais. Como resultado, tem-se solos com horizontes sulfúrico e petroplíntico, sendo que o ultimo reforça a influência do relevo e consequentemente mobilização de constituintes no perfil e na paisagem. Assim, sua gênese não deve ser compreendida apenas como resultado da influência do material de origem in situ, mas sim a partir de uma redistribuição numa concepção pedogeomorfológica.

Considerando as condições antárticas, a Península Barton apresenta intemperismo químico significativo em áreas de solos sulfatados, incluindo solos que estão entre os mais ácidos encontrados na Antártica Marítima. Estes ambientes apresentam paisagens singulares e geoformas poligenéticas, demonstrando que a Antártica reprersent um laboratorio de destaque para estudos pedológicos.

\section{REFERÊNCIAS}

ARMSTRONG, D. C. Acid Sulphate Alteration in a Magmatic Hydrothermal Environment, Barton Peninsula, King George Island, Antarctica. Mineralogical Magazine, v. 59, n. 396, p. 429-441, 1995

BEYER, L.; BÓLTER, M. Geoecology of Antarctic Ice-Free Coastal Landscapes. Springer- Verlag, Berlin Heidelberg, 2002. $420 \mathrm{p}$

BOCQUIER, G. Génèse et évolution de deux toposéquences de sols tropicaux du Tchad: intérpretation biogéodynamique. Mém.ORSTOM, v. 62, 1973. 351 p

BRINDLEY, G.W.; BROWN, G. Crystal Structures of Clay Minerals and Their X-ray Identification (Monograph 5). Min. Soc., London, 1980. 495 p.

CAMPBELL, I. B.; CLARIDGE, G. G. C. Antarctica: soils, weathering processes and environment. Amsterdam: Elsevier, 1987. 367 p.

EMBRAPA - Empresa Brasileira de Pesquisa Agropecuária. Centro Nacional de Pesquisa de Solos, Manual de Métodos de Análise de Solos. 2.ed. Rio de Janeiro, Embrapa, 1997. 212.p.

FRANCELINO, M. R.; SCHAEFER, C. E. G. R.; SIMAS, F. N. B. FERNANDES FILHO, E. I.; SOUZA, J. J. L. L.; COSTA, L. M. Geomorphology and soils distribution under paraglacial conditions in an ice-free area of Admiralty Bay, King George Island, Antarctica. Catena, v. 85, n. 3, p. 194-204, 2011. 
GOUDIE, A. Duricrust in tropical and subtropical landscapes. Oxford: Clarendon Press, 1973. 174p.

IUSS WORKING GROUP WRB. World reference base for soil resources 2006. [s.l: s.n.]. v. 43145 p.

LEE, Y. IL; LIM, H. S.; YOON, H. IL. Geochemistry of soils of King George Island, South Shetland Islands, West Antarctica: Implications for pedogenesis in cold polar regions. Geochimica et Cosmochimica Acta, v. 68, n. 21, p. 4319-4333, 2004.

LÓPEZ-MARTINEZ, J.; SERRANO, E.; SCHMID, T.; MINK, S.; LINÉS, C. Periglacial processes and landforms in the South Shetland Islands (northern Antarctic Peninsula region). Geomorphology, v. 155156, p. 62-79, 2012.

McFARLANE, M.J. Laterite and Landscape. London: Academic Press, 1976. 151 p.

MICHEL, R. F. M.; SCHAEFER, C. E. G.R; LÓPEZ-MARTINEZ, J.; SIMAS, F. N. B.; HAUS, N. W.; SERRANO, E.; BOCKEIM, J. G.. Soils and landforms from Fildes Peninsula and Ardley Island, Maritime Antarctica. Geomorphology, 225, p.. 76-86, 2014.

SERRANO, E.; LOPEZ-MARTINEZ, J. Rock glaciers in the South Shetland Islands, Western Antarctica. Geomorphology, v. 35, n. 1-2, p. 145-162, 2000.

SIMAS, F. N. B.; SCHAEFER, C. E. G. R.; MELO, V. F.; GILKES, R. J.. Clay-sized minerals in permafrost-affected soils (Cryosols) from King George Island, Antarctica. Clays and Clay Minerals, v. 54, n. 6, p. 721-736, 2006.

SIMAS, F. N. B.; SCHAEFER, C. E. G. R.; ALBUQUERQUE FILHO, M. R.; FRANCELINO, M. R.; FERNANDES FILHO, E. I.; COSTA, L. M.. Genesis, properties and classification of Cryosols from Admiralty Bay, maritime Antarctica. Geoderma, v. 144, n. 1-2, p. 116-122, 2008.

SOIL SURVEY STAFF. Keys to soil taxonomy. Soil Conservation Service, v. 12, p. 410, 2014.

SOUZA, K. K. D.; SCHAEFER, C. E. G. R.; SIMAS, F. N. B.; SPINOLA, D. N.; DE PAULA, M. D.. Soil formation in Seymour Island, Weddell Sea, Antarctica. Geomorphology, 225, p. 87-99, 2014.

TARDY, Y. Pétrologie des latérites et des sols tropicaux. Masson, Paris, 1993. 535p.

YEO, J. P. LEE, J. Ik.; HUR, S. D.; CHOI, B.G. Geochemistry of volcanic rocks in Barton and Weaver peninsulas, King George Island, Antarctica: Implications for arc maturity and correlation with fossilized volcanic centers. Geosciences Journal, v. 8, n. 1, p. 11-25, 2004.

YEOMANS, J. C.; BREMNER, J. M. A rapid and precise method for routine determination of organic carbon in soil 1. Communications in Soil Science and Plant Analysis, v. 19, n. 13, p. 1467-1476, 1988.

YOON, H. I. PARK, B. K.; KIM, Y.; KIM, D. . Glaciomarine sedimentation and its paleoceanographic implications along the fjord margins in the South Shetland Islands, Antarctica during the last 6000 years.

Palaeogeography, Palaeoclimatology, Palaeoecology, v. 157, p. 189-211, 2000. 